\title{
Polysèmes
}

Revue d'études intertextuelles et intermédiales

\section{Made in Britain (Grande-Bretagne, 1983) : blanc, masculin, violent - un portrait de la Grande- Bretagne des années 1980}

\section{Andrea Grunert}

\section{OpenEdition}

\section{Journals}

Édition électronique

URL : http://journals.openedition.org/polysemes/1672

DOI : $10.4000 /$ polysemes. 1672

ISSN : 2496-4212

Éditeur

SAIT

Édition imprimée

Date de publication : 1 janvier 2005

Pagination : 167-184

ISSN : 0999-4203

\section{Référence électronique}

Andrea Grunert, « Made in Britain (Grande-Bretagne, 1983) : blanc, masculin, violent - un portrait de la Grande-Bretagne des années 1980 », Polysèmes [En ligne], 7 | 2005, mis en ligne le 01 janvier 2005, consulté le 03 mai 2019. URL : http://journals.openedition.org/polysemes/1672 ; DOI : 10.4000/ polysemes. 1672

Ce document a été généré automatiquement le 3 mai 2019.

Polysèmes 


\title{
Made in Britain (Grande-Bretagne, $\left.1983^{1}\right)$ : blanc, masculin, violent - un portrait de la Grande-Bretagne des années 1980
}

\author{
Andrea Grunert
}

1 Bien qu'il soit considéré comme un auteur à part entière, Alan Clarke (1935-1990) et son œuvre restent à découvrir. Ceci est en large partie dû au fait que la plupart des court-, moyen- et long-métrages qu'il a tournés depuis le début des années soixante-dix ont été réalisés pour la télévision, notamment pour la BBC. Cependant, sans le concours de cet autre médium, une partie non-négligeable de la production des films en Grande-Bretagne n'aurait pas été possible 2 . Depuis sa mort en 1990, les films de Clarke ont été montrés à maints festivals dont celui d'Edinburgh qui lui a consacré une rétrospective en 1998. Alan Clarke est désormais une figure-clef du cinéma britannique ${ }^{3}$. Son œuvre et sa manière de travailler continuent à influencer des acteurs et des metteurs en scène comme le témoignent les films réalisés récemment par Gary Oldman ${ }^{4}$ et Tim Roth ${ }^{5}$ qui ont tous les deux joué dans des films de Clarke.

2 Tout comme Ken Loach ou Mike Leigh, Clarke fait partie de ces réalisateurs anglais dont les films révèlent un intérêt profond pour l'être humain et le social. Partant des traditions plus anciennes du cinéma anglais et de ses conventions réalistes ${ }^{6}$, ses films développent une approche artistique tout à fait individuelle centrée sur la condition humaine. La filmographie de Clarke qui comprend des adaptations pour la télévision de La Mort de Danton de Georg Büchner (1978) et de Baal de Bertolt Brecht (1982) présente une trop grande variété pour se laisser réduire à un seul thème. Néanmoins, parmi ses réalisations les plus marquantes, on compte les films qui cherchent à sonder les mentalités et les identités anglaises très souvent mises en rapport avec la violence. Scum (1977; version pour le cinéma de 1979), Made in Britain (1983), Contact (1984), Christine (1986), Road (1987), Elephant (1988) ou The Firm (1988) traitent de la situation dans un centre de redressement pour adolescents, de la délinquance juvénile, de la violence en Irlande du Nord, du trafic 
de drogue et de la vie quotidienne des défavorisés de la société contemporaine en GrandeBretagne. Les protagonistes de ces films qui font le constat d'une société malade et d'un système social défaillant sont des hooligans, des skinheads racistes, des sans-abris, des chômeurs. Présentant des personnages principaux plutôt inhabituels, les films de Clarke créent des modèles opposés aux images optimistes propagées par le gouvernement Thatcher.

\section{Actions violentes}

3 Pénétrant au cœur de la violence, du racisme et de la privation, Made in Britain s'intègre à un ensemble de films au cœur d'une filmographie qui propose de manière quasiment obsessionnelle des réflexions sur les formes de l'agressivité dans une société hypocrite qui la condamne tout en la provoquant. Ce film réalisé pour la télévision ${ }^{7}$ nous apparait comme un exemple particulièrement éloquent permettant de montrer les préoccupations thématiques de Clarke et sa démarche formelle. Notre approche consistera en une analyse approfondie des éléments thématiques, narratifs, figuratifs et esthétiques mis en place par le film de Clarke. À partir de l'exemple filmique, nous chercherons à dévoiler les diverses formes que la violence revêt dans Made in Britain et à aboutir à des réflexions sur la manière qu'a le réalisateur d'envisager la violence et son rôle dans la Grande-Bretagne actuelle.

Dans le texte filmique dont nous allons traiter la violence est présente dans le récit et elle est représentée dans et par l'image. Apparaissant sous forme d'actes, d'actions et d'attaques verbales, elle détermine les relations interpersonnelles mises en scène. Pourtant, Clarke ne l'investit pas uniquement sur le plan thématique et narratif. Au contraire, il a recours à tout un répertoire de codes cinématographiques qui sont générateurs et vecteurs des effets de violence. La figure de la violence traduite par des éléments plastiques et repérables dans l'expérience perceptive introduit des basculements, des fissures et des ruptures qui participent à l'organisation des composantes du récit filmique. Étant au cœur même de ses moyens d'expression, la violence n'est pas seulement présente à l'image, mais elle pénètre la texture du film.

5 Le protagoniste de Made in Britain est Trevor (joué par Tim Roth), un jeune skinhead de seize ans dont le film relate l'itinéraire autodestructeur, l'arrivée au centre de redressement et de réhabilitation, son bref séjour et sa fuite qui sont des successions d'actes violents et sa nouvelle arrestation. Autour de ce personnage central, le réalisateur multiplie les aperçus sur la violence et ses formes d'expression ainsi que sur ses agents. Trevor est envoyé au centre correctionnel pour plusieurs délits dont vol, vol à l'étalage et pour avoir cassé la vitre de la maison d'un Pakistanais. Par la suite, on le montre en train de commettre d'autres actes violents : il détruit la vitrine de l'agence de l'emploi et, une seconde fois, il casse des vitres chez le Pakistanais et chez ses voisins. Parce qu'on lui refuse le repas à cause de sa rentrée tardive au centre, il enfonce une porte et maltraite le cuisinier à coups de poing et de pied.

C'est pourtant la seule fois où il commet un acte de violence aussi grave contre un être humain. De manière générale, la violence de Trevor se manifeste contre des objets. Ainsi urine-t-il sur les dossiers au centre et persuade-t-il Errol, un autre adolescent, de déféquer sur d'autres dossiers. À part la violence physique, Trevor a encore plus souvent recours à la provocation verbale. Il traite tout le monde de wankers et écrit le mot même sur le tableau noir de la pièce où on l'avait enfermé après sa bagarre à la cantine. Les 
cibles de ses attaques verbales sont le système social établi et ses représentants. L'autre objet de sa haine sont les étrangers qu'il traite de Pakis, de niggers ou encore de nignogs. « I am British. Pakis don't even speak fucking English ", dit-il. Clarke associe le skinhead à un discours raciste et à l'extrême droite, comme la croix gammée qu'il s'est fait tatouer sur le front le signale ${ }^{8}$. Le racisme explique en partie ses actes de vandalisme; les étrangers deviennent la soupape de ses frustrations.

7 L'attitude du protagoniste est dominée par l'agressivité de manière que la violence verbale et physique remplacent d'autres formes d'expression ou d'émotion et deviennent ainsi l'ersatz d'une communication positive. Tous les rapports sociaux du skinhead semblent être négatifs. Il entraîne Errol dont il est devenu l'objet d'admiration dans son jeu violent. Ensemble ils se rendent dans le quartier pakistanais pour y casser des vitres et lancer des propos racistes. Errol, le Noir, imite sa nouvelle idole, mais, surtout, il subit et supporte les propos racistes et l'attitude méprisante de Trevor qui abuse de son intelligence supérieure. Il en est de même pour le jeune homme dans l'agence pour l'emploi, apparemment illettré, dont Trevor se moque tout en visant un système social incapable de lui fournir un travail adéquat. À la fin, Trevor dépose la voiture volée devant un poste de police et abandonne Errol qui s'est endormi dans ladite voiture. Ce n'est que pendant une course avec des vieilles voitures qu'il semble éprouver une certaine joie. Encore une fois il s'agit d'un moment de compétition qui n'est pas dépourvu de violence et qui exige une manière de conduire très agressive $e^{9}$.

8 Se concentrant sur ce personnage, le récit linéaire de Made in Britain se présente comme une structure ponctuée de moments violents. L'itinéraire du skinhead se transforme en une folle poursuite où la colère, l'agressivité et les frustrations s'enchainent et se nourrissent les uns les autres. Cette violence va s'accroître et finalement se retourner contre l'adolescent. Après avoir enfoncé la porte de la cantine, trois hommes emmènent Trevor qui se débat. À la fin, on le voit dans la cellule d'un poste de police où il ne cesse de provoquer les deux policiers. À ses provocations, l'un des agents répond en le frappant brutalement avec un bâton.

\section{Mouvements sans issue}

Outre qu'il représente les actions violentes et les expressions agressives de différents personnages, Clarke cherche à créer un sentiment de tension et de violence par les moyens techniques et esthétiques mis à sa disposition. Tous les moyens de l'expression du cinéma participent à la représentation de la violence, les éléments visuels autant que les éléments sonores et la musique, ou encore, l'absence de celle-ci. L'un de ces moyens d'expression essentiels concerne le jeu des acteurs, en particulier celui de l'acteur principal. Les images autant que le jeu des acteurs évoquent les blessures, les fissures et les ruptures qui informent le social. Le programme actoral ${ }^{10}$, le jeu et le corps de l'acteur et la lecture diégétique se complètent : espaces visuels et caractère/corps révèlent tous les deux de la violence. Que Trevor se trouve au centre de ce discours filmique, le jeu sans compromis de Roth ne fait que le souligner. Le goût pour la violence est inscrit sur le corps du protagoniste. Le crâne rasé, les bras et le torse tatoués, il porte l'uniforme des skinheads et son corps ainsi mis en scène affiche ses convictions et dégage sa volonté d'agression. Le corps devient signe avant la lettre, signifiant par lui-même. Mimique et langage corporel de l'acteur le font apparaitre comme une bombe à retardement humaine, prête à exploser à tout moment. Le corps raide et tendu, exprimant une colère à 
peine retenue, les mouvements brusques, le visage déformé par la haine: à chaque moment, Roth présente le portrait d'un adolescent possédé par la rage et les frustrations dont le comportement violent et finalement autodestructeur semble être la seule perspective de vie.

10 À la fois ouvertement exprimée et intériorisée, la représentation de la violence n'est ni camouflée par le spectacle et le spectaculaire comme dans le cinéma d'action, ni liée à la volonté d'offrir de l'évasion. Élément de structure, elle n'est pas utilisée pour créer du suspense. Omniprésente, cette violence monte à la surface de la pellicule dans un film où tous les éléments concourent à la rendre visible et tangible. La tension violente qui traverse Made in Britain d'un bout à l'autre se manifeste moins à un niveau physique que de manière souterraine. Dans des films comme Contact, Christine ou Elephant, Clarke cherche à faire surgir un sentiment de violence et d'oppression par une approche particulière de la notion du temps. La durée des plans, la répétition de mouvements et d'actions reproduisent la routine quotidienne, l'ennui, la banalisation, tous générateurs de frustrations. Conforme à un sentiment pessimiste, le récit filmique de Made in Britain reprend la forme circulaire, traduite en cercle vicieux pour son protagoniste, où même les détails - les plans, les situations particulières et les dialogues - participent à la mise en scène de l'idée d'une existence sans issue dont le protagoniste est la victime.

11 L'image du cercle, du mouvement sans issue et de fermeture est exprimée dans la séquence où l'on voit Trevor enfermé dans une pièce après avoir attaqué le cuisinier. Le jeune homme commence à marcher en rond. Répétant ce mouvement, il apparait comme un animal dans sa cage qui ne réussit pas à canaliser l'énergie qui le possède. Une telle existence lui est prédite par l'inspecteur de police qui lui explique son destin en en faisant même une esquisse sur le tableau noir: sans formation et sans travail, il va finir par commettre des vols, le vol le mènera en prison, après avoir fait de la prison, il va se trouver de nouveau sans travail et sans argent et le cercle infernal recommencera. La vision qui en découle traverse le film entier et lui donne sa structure : à chaque acte violent de l'adolescent, le système social répond par des moyens de contrôle et de punition tout aussi violents. À la fin, la boucle semble bouclée : Trevor est de nouveau arrêté. Le déclenchement abrupt de la même musique punk qui accompagne sa première apparition à l'écran signale que son itinéraire violent va continuer et souligne qu'il est toujours en révolte.

12 Clarke crée des espaces physiques, sociaux, mentaux et psychiques limités. Si la musique punk ajoute encore une note agressive à l'allure provocatrice du personnage principal, c'est surtout l'absence générale de musique qui augmente le sentiment d'oppression émergeant dans le film. Cadrages, angles de prise de vue et mouvements de caméra font naître des fermetures visuelles; la caméra qui colle aux personnages limite leur champ d'action. Les procédés de fragmentation qui comprennent l'utilisation d'un éclairage minimal et des couleurs sombres et sales contribuent à l'ambiance de claustrophobie. Une atmosphère similaire se dégage de la mise en scène des espaces. La violence marque les paysages urbains sordides dont le film ne livre que quelques rares vues. Ce sont des lieux publics anonymes: des couloirs, un tunnel, des bureaux, une piscine désaffectée, une école en briques sombres dont Trevor longe la double grille d'entrée. De telles images et l'accumulation de séquences qui se déroulent dans des intérieurs créent l'idée d'oppression et de fermeture.

13 D'autres stratégies textuelles - de la mise en scène et du montage - font naître un sentiment d'instabilité. Les légers basculements de la caméra mobile articulent l'idée de 
la perte d'identité aussi bien que les gros plans obliques le suggèrent par les vues incomplètes du visage de Trevor montré donc de trois-quarts. Une tension émerge de ces gros plans qui accentuent l'isolement du protagoniste et son aliénation. Un choc visuel est créé quand, dans la première séquence, le spectateur est soudain confronté avec le corps du protagoniste qui se lève et commence à marcher en direction de la caméra comme s'il était projeté en avant. Les procédés de fragmentation et les changements brusques de la mobilité à l'immobilité deviennent générateurs d'une violence sourde, latente et de conflit permanent. Clarke crée des espaces émotionnels multiples où le spectateur est exposé à des expressions de violence immédiate tout comme il est intégré dans le corps du film. Bien que Trevor ne regarde pas directement la caméra, dans certains gros plans de son visage où il crache sa haine, son hostilité semble se propager non seulement aux personnages hors-champ, mais aussi au spectateur hors écran. Au centre de la représentation et des réflexions filmiques sur la violence, la présence du corps $\mathrm{du}$ protagoniste est articulée dans son rapport au spectateur. Clarke instrumentalise le corps de son acteur principal sans le réduire à un simple vecteur du récit. Le corps de Trevor/Roth s'offre au spectateur, à ses fantasmes, ses angoisses, sa colère. Centrifuge et centripète, la violence subie et distribuée par le protagoniste est renvoyée au spectateur qui subit ses effets ${ }^{11}$.

Le corps de Trevor dominant parfois l'espace est aussi un corps traqué et objectifié, exposé au regard de la caméra et à celui du spectateur qui l'observe à travers une vitre (qui double la vue à travers l'objectif et le cadre) quand, enfermé dans une pièce, il tourne en rond sans but. Par moments, le spectateur se transforme en voyeur ${ }^{12}$, sans que Clarke lui accorde la possibilité de la délectation, car son statut de voyeur est immédiatement détourné et rendu explicite. L'idée de la violence associée au regard produit donc constamment des effets et des contre effets troublants. Il en est de même quand Errol cherche encore une fois - bien qu'inconsciemment - à surpasser son idole. La séquence où il hurle « Go back to the jungle ! » à l'adresse des Pakistanais n'est pas dépourvue d'ironie. La conscience du spectateur qu'Errol lui-même serait l'objet habituel de tels propos le confronte avec ses propres conventions racistes.

Produit pour la télévision ${ }^{13}$, Made in Britain projette sa violence par un médium dont les images accompagnent la vie quotidienne de la plupart d'entre nous comme une vie parallèle. Les techniques de la télévision et du documentaire s'ajoutent à celle de la caméra mobile qui offre une impression d'immédiateté. Pourtant, l'emploi de la steadicam, de la caméra portée, et les changements brusques du mouvement à l'arrêt, de l'inactivité à l'action, de l'attachement au détachement rendent ouvertes les techniques du cinéma et de la télévision. Engendrant une distance formelle, ils introduisent aussi des moments de choc et de tension, nés des mouvements de déséquilibre entre émotion et réflexion.

\section{Espaces dominés}

16 Si le sentiment d'oppression et de fermeture est inévitable, Trevor domine l'espace filmique et dramatique tout comme il domine le récit. Tout d'abord par sa présence presque constante dans l'image. Tous les moyens de la mise en scène sont mis en place afin de le mettre au centre du récit filmique. Le gros plan en est un, l'utilisation de la steadicam en est un autre. La caméra capte Trevor pendant ses longues marches dans les couloirs, les rues, le tunnel et s'attarde sur lui. Les longs travellings qui l'accompagnent 
ou le suivent ont aussi des fonctions expressives et sémantiques signifiant la résistance et l'énergie du personnage.

Le film montre clairement que les mouvements d'évasion de Trevor sont illusoires. En même temps, le jeu de Roth fait apparaître un jeune homme sûr de lui-même qui - à tort ou à raison - ne cesse de revendiquer ce qu'il pense être ses droits. Libéré de la pièce dans laquelle on l'avait enfermé, il se trouve au deuxième plan de l'image, un peu en retrait de Peter Clive, le directeur du centre, et de Barry, l'un de ses collaborateurs, placés au premier plan à sa gauche et à sa droite. Cependant, cette fermeture visuelle ne crée pas le même sentiment d'oppression, voire d'écrasement que dans Scum où Clarke l'utilise à plusieurs reprises à de telles fins. Car, ici, Trevor répond aux adultes par un sourire moqueur comme s'il voulait dire «je vous ai bien eus ». Il contrôle et domine l'espace qu'il traverse à grandes enjambées et par son attitude décidée. Très subtilement, Clarke introduit le sentiment que le jeune homme contrôle aussi son entourage. Dans les couloirs de la cour ou dans ceux du centre de redressement et de réhabilitation, c'est lui qui indique la vitesse pendant que Harry, le travailleur social, est obligé de s'adapter à son rythme.

La tension qui habite Trevor et qu'il dégage possède une composante psychologique. Elle est l'expression de l'aliénation du personnage et une forme de résistance à l'égard de la réintégration normative exigée par les travailleurs sociaux, les enseignants, les policiers. Sa colère est impuissante, certes. C'est pourtant son agressivité qui fait de Trevor un individu, dont l'identité est révélée dans la confrontation et le conflit. Son énergie qui frôle l'hyperactivité est destructrice et nihiliste, mais signale aussi qu'il est vivant. Par son attitude provocatrice, il met le policier hors de lui. «L'acte de provocation est une mise à l'épreuve ", écrit Jean-Claude Arfouilloux ${ }^{14}$, dans ce cas, une mise à l'épreuve des limites : Trevor apparaît comme un enfant qui teste les limites de l'adulte, mais qui, cette fois-ci, a mal calculé l'enjeu, car la réaction brutale de l'agent de police qui le terrasse le surprend. Cependant, dans le dernier plan du film, la caméra se fige sur son visage où l'expression de la douleur et de l'étonnement est remplacée par un sourire à la fois moqueur et triomphant. Le jeune skinhead est à terre, certes, mais il n'est pas brisé.

\section{Points de vue}

Made in Britain offre des possibilités d'identification avec son personnage principal autant que le film crée de la distance critique. En fait, il s'agit d'un type d'identification qui fonctionne comme le processus textuel de la relation entre le personnage et le spectateur, «by which spectators are placed in relation to characters in terms of access to their actions and to what they know and feel $»^{15}$. Clarke livre un portrait hautement ambigu du skinhead où le refus et la fascination se mélangent. Loin d'en faire un personnage sympathique ou attrayant, son film cherche à amener le spectateur à comprendre ses frustrations.

La participation spectatorielle et la manière dont le spectateur est inscrit dans l'espace filmique se présentent sous des facettes variées. Les longs travellings de Trevor, où la caméra semble s'accrocher à lui, entraînent le regard du spectateur sans lui offrir d'échappatoire. Comme Howard Schuman l'a écrit : «The viewer is borne along helplessly as Trevor marches on his rounds: whether he's destroying a job centre or smashing the windows of Asian homes, the viewer becomes his accomplice $»^{16}$. Le spectateur est ainsi 
placé en position de témoin, confronté aux effets de violence produits par l'action et les moyens d'expression déployés.

21 Trevor s'impose par ses mouvements et par son regard. Le film de Clarke, par le biais de son organisation de l'espace et par le montage, met l'accent sur la subjectivité du point de vue. Dans l'une des séquences-clefs, la discussion violente qui oppose le skinhead à Peter Clive et Barry, le point de vue de Trevor, étant le foyer de vision dans une structure de plans alternants, oriente la perspective narrative. Une échelle d'importance des deux interlocuteurs du protagoniste est visiblement créée par le cadrage et par les angles de prise de vue. Trevor adresse ses propos violents davantage à Barry, l'homme plus jeune et plus agressif que Clive, que l'adolescent réussit à provoquer plus facilement. Quant au directeur du centre, il est visiblement débordé par la situation et ne semble pas vraiment compter pour le skinhead qui le méprise.

\section{Sans réponses}

22 Trevor qui définit sa Britishness par des propos racistes fait partie de ces personnages qui n'ont guère de porte-parole. En partant de clichés, le portrait du jeune skinhead échappe pourtant à l'imagerie habituelle. Loin d'être une brute, il est un adolescent à l'esprit et à l'œil vifs, intelligent et habile. Clarke dénonce son attitude raciste, mais il le présente comme étant à la fois un malfaiteur et une victime. Si Trevor échappe à la représentation conventionnelle de la victime, c'est Errol qui remplit ce rôle en inspirant la pitié ${ }^{17}$. Trevor refuse de se faire aider et semble n'avoir aucune conscience de son attitude anti-sociale et criminelle («Are you ashamed? », demande le juge et Trevor répond « No.»), il est, au contraire, manipulateur et provocateur : il n'a rien de la passivité d'une victime. Bien au contraire, le film célèbre l'énergie de son protagoniste tout en dévoilant par moments sa vulnérabilité et en faisant entrevoir que son assurance n'est que factice.

Les propos de Trevor se composent de préjugés, de clichés, de généralisations. Ainsi reproche-t-il aux immigrés de prendre leur travail aux Anglais. Quand il lit une petite annonce où un emploi est offert à une personne parlant couramment l'ourdou et le pendjabi, ses arguments semblent soudain avoir une certaine part de vérité. Parce qu'il ne commente pas cette situation, le metteur en scène se dérobe un peu trop facilement. Cependant, il ne justifie pas l'attitude de son protagoniste, mais souligne de nouveau ses frustrations.

Made in Britain - ce titre résonne comme un label désignant des produits tels que le racisme et la délinquance juvénile, en fait, des phénomènes que la société dépeinte dans le film a du mal à maîtriser. Dans la mesure où il n'explique pas le comportement agressif de Trevor, Clarke ne réduit pas les problèmes de la violence et du racisme à une simple relation de cause à effet entre le jeune homme et la société bien que le film suggère parfois un tel lien ${ }^{18}$. Le réalisateur détecte les signes de vie et les symptômes de ratage. Se contentant de faire des constats, de montrer des actions et des réactions, il se refuse aux engagements et aux interprétations faciles. De même qu'il évite une approche didactique et les grandes oppositions manichéennes qui structurent beaucoup de films américains, en se concentrant sur les attitudes des êtres humains.

Dans Made in Britain, Clarke met le doigt sur une plaie et met une société figée face à ses problèmes. En présentant le jeune skinhead comme étant le prisonnier d'un système incompétent et de sa propre démarche autodestructrice, le réalisateur évite d'introduire 
un point de vue moralisateur et se refuse à condamner d'emblée son personnage principal. Au contraire, il confronte son discours raciste à un système sans réponses véritables et à son argumentation elle-même stéréotypée. Au comportement défendant la norme du protagoniste, Clarke oppose un système éducatif et correctionnel auquel il reproche son inefficacité et son hypocrisie. Ce système reconnaît sa propre inefficacité quand, par exemple, l'inspecteur de police dévoile que la prison n'est pas un lieu qui mène à la resocialisation mais un lieu qui contribue à la délinquance ${ }^{19}$. Aux adultes qui ne peuvent que lui tracer son itinéraire futur sur un tableau noir, Trevor répond : « Teachers don't want kids to be honest, they'd lose control... all school teaches you is to work hard and get a job otherwise you're no good». Si Clarke ne nie pas que son protagoniste ne cesse de provoquer et rejette tout geste de secours, il oppose l'adolescent à une société qui est incapable de lui offrir une perspective véritable et ne lui propose que des petits boulots mal payés.

Son film suggère que la violence est partie intégrante d'un système judiciaire de correction, de réhabilitation, de punition. Made in Britain montre en filigrane les moyens de pouvoir déployés par le système social et idéologique en place : la marginalisation, voire l'exclusion et la réadaptation, voire l'intégration sociales suivant les normes imposées. Plus encore, le film présente une société effrayée par des adolescents comme Trevor et des adultes qui se sont résignés ou se contentent de gérer des cas. Si Harry et Peter Clive témoignent d'une certaine bonne volonté envers le skinhead, Barry pense que s'occuper de Trevor constitue une perte de temps. Ceci paraît cynique mais, compte tenu du comportement provocateur et agressif de Trevor qui se refuse à tout dialogue, une telle opinion reste ambivalente. À côté des pédagogues et travailleurs sociaux frustrés, Clarke présente, sous les traits de Peter Clive, un homme qui se veut tolérant, mais qui est visiblement débordé par sa tâche. Objet du dédain et cible de l'impertinence de Trevor qui réussit à lui voler son trousseau de clefs et l'empêche ainsi de rentrer la nuit au centre dont il est le directeur, Clive est constamment ridiculisé.

À la fin, Harry livre le skinhead à la police pour protéger sa vie privée et sa famille et, surtout, son propre confort. Dans cette séquence où Trevor fait intrusion dans l'espace intime du travailleur social, Clarke ne présente pas son protagoniste comme une victime qui réclame de la sympathie, mais comme un garçon qui, de manière insolente, se vante de ses exploits violents. Il apparait comme l'incarnation parfaite des problèmes que tout le monde préfère éviter. Là encore, le réalisateur confronte Harry et le spectateur aux limites de leur tolérance, une attitude de tolérance qui accepte plus facilement le mendiant humble que celui qui refuse l'adaptation sociale. Trevor est tenu à l'écart de la famille apparemment intacte de Harry. Alors qu'il contemple les deux enfants de Harry dormant dans leur chambre, celui-ci le fait sortir brusquement. Si le travailleur social est peut-être la seule personne à qui le skinhead fait encore confiance, il va le décevoir et appeler la police. Le bonheur familial reste donc inaccessible pour le jeune homme. Il se révèle être une pure fabrication, un décor figé dans la vitrine d'un magasin où quatre mannequins de cire imitent une scène familiale. L'adolescent regardant longuement ce dispositif de la famille parfaite en est autant exclu que de la famille en chair et en os. La mise en image de l'idée de l'exclusion fonctionne à double tranchant et évoque toute une problématique sociale ${ }^{20}$. Elle fait allusion à une société qui refuse à une partie de la population et à certains jeunes la participation aux biens sociaux, culturels et économiques. En même temps, elle présente un adolescent que la colère permanente empêche de développer des facultés sociales. 
et le jeune homme dans l'agence de l'emploi sont analphabètes ou quasianalphabètes. Dans Made in Britain, ces «tales out of school " ${ }^{21}$, les adolescents sont des laissés-pour-compte dans un système social et éducatif en faillite qui, à la fin, répond aux provocations du skinhead par une violence démesurée, annonciatrice d'une autre spirale de violence sans fin et encore renforcée. Le sentiment de violence est inhérent au constat de l'échec et à la représentation de la crise - de l'individu, du social - faits par Clarke. Jamais gratuite, la mise en scène de la violence cerne les conditions de vie d'une certaine jeunesse en Grande-Bretagne. Sans donner des réponses claires et sans proposer de solutions, Made in Britain se termine sur un dilemme non résolu et n'offre aucun soulagement.

\section{NOTES}

1. Réalisation : Alan Clarke. Scénario : David Leland. Caméra : Chris Menges. Montage : Stephen Singleton. Productrice : Margaret Matheson. Production : Central Television. Interprètes : Tim Roth, Bill Stewart, Eric Richard, Geoffrey Hutchings, Sean Chapman. Couleur. 78 min.

2. Comme l'a écrit Philippe Pilard à l'égard de la production de films en Angleterre pendant les années 1980: «Dans le monde audiovisuel britannique, la télévision joue donc un rôle primordial. Et l'on ne peut guère comprendre certains phénomènes ou événements du cinéma, si l'on ignore tout de la télévision. " (Le Nouveau cinéma britannique 1979-1988, Renens (CH), Foma/5 Continents, 1989, 66.) Parmi les metteurs en scène qui ont réalisé des films pour la télévision ou avec son aide on peut compter Ken Loach, Mike Leigh, Stephen Frears, Michael Radford, David Hare et bien d'autres.

3. Voir pour le rôle de Clarke dans le cinéma britannique : Justine Ashby et Andrew Higson eds., British Cinema, Past and Present, London, Routledge, 2000, 248.

4. Nil by Mouth, Grande-Bretagne, 1997.

5. The War Zone, Grande-Bretagne, 1999.

6. Voir John Hill, «From the New Wave to "Brit-Grit": Continuity and Difference in Working Class Realism », in J. Ashby and A. Higson eds., British Cinema, Past and Present, op. cit., 249-260.

7. Made in Britain a été réalisé pour Central Television qui fait partie du réseau ITV, une chaîne privée établie en 1954.

8. II faut mentionner qu'à ses origines, le mouvement des skinheads était ancré dans la culture des ouvriers anglais où il a pris naissance à la fin des années 1960 sans avoir été motivé par le racisme et une tendance particulière vers l'extrême droite.

9. Plaisir et violence faite à son propre corps vont de pair quand Trevor renifle de la colle.

10. Nous empruntons ce terme à Nicole Brenez qui le définit dans son article « La nuit ouverte: Cassavetes, l'invention de facteur ", dans Le Théâtre dans le cinéma, Paris, Cinémathèque française, 1992-93, 89-102.

11. Pour les réflexions théoriques sur l'expérience filmique en tant que structure expressive et perceptive et les activités dynamiques impliquées dans cette structure qui se rapportent au réalisateur, au film et au spectateur, on peut se référer par exemple à Vivian Sobchack, «Phenomenology and the Film Experience » dans Linda Williams, ed., Viewing Positions: Ways of Seeing Film, New Brunswick, N.J., Rutgers UP, 1995, 36-58. 
12. Nous définissons le terme "voyeur " ici au sens large en tant que "the iconic, postmodem self. Adrift in a sea of symbols, we find ourselves, voyeurs all, products of the cinematic self.» (Norman K Denzin, The Cinematic Society: The Voyeur's Gaze, London, Sage, 1995, 1).

13. La réception spectatorielle d'un film diffusé par la télévision est différente de celle dans une salle de cinéma et la différence de format et de perception influe sur certains aspects de la mise en scène. Si ce facteur intervient dans ce texte, c'est qu'il pose ici un faux problème qui est encore souligné par le fait que les films de Clarke, d'abord destinés à l'exploitation télévisuelle, passent aujourd'hui sur le grand écran où les différences semblent pourtant s'effacer.

14. «Provocations ", La Nouvelle Revue de Psychanalyse, XXXI, printemps 1985, 141.

15. Murray Smith, Engaging Characters: Fiction, Emotion and the Cinema, Oxford, Clarendon Press, 1995, 123. Smith n'emploie pas le terme « identification », mais propose celui d'alignment.

16. « Alan Clarke: In It for Life », Sight and Sound, September 1998, 20.

17. Même quand il frappe, il fait pitié, ce que la séquence de l'attaque dans le quartier pakistanais et celle où Trevor l'abandonne devant le poste de police renforcent.

18. À la mise en scène des individus en proie à l'aliénation, il associe les structures sociales en décomposition. L'espace social ainsi dépeint par le réalisateur crée un contre-courant à l'idéologie de la famille associée au thatcherisme. Il présente des variations parodiques et dérisoires de la famille (la famille idéale représentée par les mannequins de cire dans la vitrine d'une grande surface) ou - dans le dialogue - fait allusion aux structures familiales éclatées. Cependant, il ne cherche pas à approfondir ces allusions ni sur le plan dramaturgique ni sur le plan de la réflexion sociale.

19. Ce que Michel Foucault exprime dans Surveiller et punir: la naissance de la prison, Paris, Gallimard, 1975.

20. II faut pourtant encore une fois souligner que le film de Clarke évite de faire l'anamnèse des conditions de vie de Trevor. Par contre, il est un peu plus explicite en ce qui concerne Errol qui fait sans aucun doute partie des défavorisés. De lui, on apprend qu'il est un enfant délaissé, de père inconnu et qui a été rejeté par sa mère.

21. C'est l'expression qui préfigure le film au générique.

\section{INDEX}

oeuvrecitee Made in Britain 